\title{
AN INTELLIGENT RECOGNITION SYSTEM FOR IDENTIFICATION OF WOOD SPECIES
}

\author{
Mohan, S., K. Venkatachalapathy and P. Sudhakar \\ Department of Computer Science and Engineering, Annamalai University, Annamalainagar, India
}

Received 2014-01-09; Revised 2014-02-10; Accepted 2014-02-19

\begin{abstract}
The recognition of wood species is needed is many areas like construction industry, furniture manufacturing, etc.,. The wood is traditionally classified by human experts. But human identification of wood type is not accurate and the manual identification is a time consuming process. So in this study, an intelligent recognition for identification of wood species was developed. This study uses image enhancement as a preprocessing techniques and uses a new method which divides the image into several blocks known as image blocking. Each block is extracted using grey image and edge detection techniques. The Grey-Level Co-occurrence Matrix (GLCM) is used as a texture classification technique. The GLCMs are generated to obtain three features: Entropy, standard deviation and correlation. The classification technique used to classify the wood species is correlation. Our experimental results showed that the proposed method can increase the recognition rate up to $95 \%$, which is faster and better than existing system which gives $85 \%$ recognition rate.
\end{abstract}

Keywords: Image Enhancement, Preprocessing, Wood Identification, Grey-Level Co-Occurrences Matrix, Correlation

\section{INTRODUCTION}

There are more than 15,000 indentified species of trees on the earth of which most of them are in tropical regions. With high diversity of wood species, the classification of wood species became important. Wood Classification is also important for wood-based industry. Also some of timber traders mix different types of wood to increase their profit, so identification of wood is very important.

Wood is traditionally classified by wood (human)experts. But it is impractical and cost-effective for a human to analyze and identify the wood species. Also human identification of wood type is not accurate and the manual identification is a time consuming process (Gurau et al., 2013). Human experts are not abundant in market to meet the demand in the industry. Hence automatic wood species recognition system is required and is capable of reducing the errors caused by traditional wood identification system.
All the wood species has different texture, strength, density, hardness, odor, color. Texture is one of important characteristics used in identifying the objects in an image (Hasan et al., 2013). Therefore, the classification wood species based on texture classification can be a reliable solution to solve this problem.

In previous research (Gurau et al., 2013), the system identifies the species of wood using the textural features present in its bark. The captured image undergoes preprocessing techniques. GLCM is used to retrieve the features of wood species. The features are energy, entropy, contrast, homogeneity, angular second moment. Classification is done using correlation technique.

The research conducted by the (Hazra, 2011), have designed an automatic wood recognition system using Visual System Development Platform library (VSDP) After capturing image, two procedures are used to enhance image quality using VSDP library, image sharpening and contrast enhancement. The feature extractor used is GLCM. Artificial Neural Network Corresponding Author: Mohan, S., Department of Computer Science and Engineering, Annamalai University, Annamalainagar, India 
using the back propagation algorithm is used to classify the wood.

Wood identifications are necessary in the many areas like construction industry and furniture manufacturing. In construction industry, choosing the right wood is very important mainly. In construction industry, woods are mainly used for constructing the roof truss. If the wood materials are not strong enough and used for constructing the roof truss, part of the house may collapse after a period of time. In furniture manufacturing, the type of wood used must be chosen properly for manufacturing the chairs, table and cub-boards (Bremanath, 2009). Verification of wood species is important to avoid unnecessary loss for manufactures. Identification of wood species can also be used in other areas such as:

- Determining the type of wood fragments from a crime scene

- Determining the material used in ancient architecture

- Understanding the ecological information of an area to study the relationship between the species

The primary objective of this study is to explore the possibility of developing a system which is able to perform automated wood recognition using the concept of image processing (Ardian, 2012).

This study has to achieve the following goals:

- To use a low cost equipment to identify the wood species based on its microscopic features

- To use a new method of image-based recognition system for wood type identification by dividing the wood image into several blocks

This study is organized as follows: In section II, we presented the methodology of the proposed system. In section III, experimental results are discussed followed by conclusion in section IV.

\section{PROPOSED METHOD}

At the outset of the process which is given in the Figure 1, the input images for both testing and training are acquired using a high resolution digital camera. Before the wood images are tested for its relevant species, it is mandatory for the system to be trained with all possible species available.

The data is a collection of images that has been cut into $500 \times 500$ pixels. Authenticated wood samples were obtained for testing and training the data. Before testing the images of wood, the wood images have to be trained.
For training the wood sample, image enhancement is the first step. It is done to improve the quality of the image. The image enhancement techniques used are contrast enhancement and image sharpening. Next blocking method is applied that divides the images into several blocks. RGB image blocks are converted to grey image blocks. Edge detection is applied to grey blocks. The features are extracted from grey block and Edge detected block. For each wood image, the features are extracted and stored in a separate flat file.The extracted features of a wood image are compared with the features extracted from the test image during testing process. The testing process is made very easy by identifying the correlation value between the test and the train image.

\section{WOOD SPECIES RECOGNITION}

This study can identify 10 Indian wood species namely jack, rosewood, sandal wood, satin, sal, mahogany, mulberry, oak, iron wood and teak (Tou et al., 2007).

\subsection{Image Acquisition}

The system has been set up to acquire the image of wood using high resolution digital camera. The captured image size is $1280 \times 1024$ pixels. Then image has been cropped into $500 \times 500$ pixels. This study can identify 10 Indian wood species. Each type consists of 100 images derived from wood samples. So totally there would be 1000 images. These 1000 images are used for training and testing is done by using five images of each type. Thus for testing we would have 50 images. Table 1 shows list of wood species that can be identified by this study along with their scientific name.

\subsection{Image Enhancement}

It can be defined as conversion of image quality into better and more understandable level for feature extraction. Image enhancement is the improvement of digital image quality (e.g., for visual inspection or for machine analysis) without knowledge about the source of degradation (Khalid et al., 2008).

Image enhancement will:

- Improve the interpretability or perception of information in images for human viewers

- Providing better input for other automated image processing techniques

In this study, contrast enhancement and sharpening is used as image enhancement techniques. 


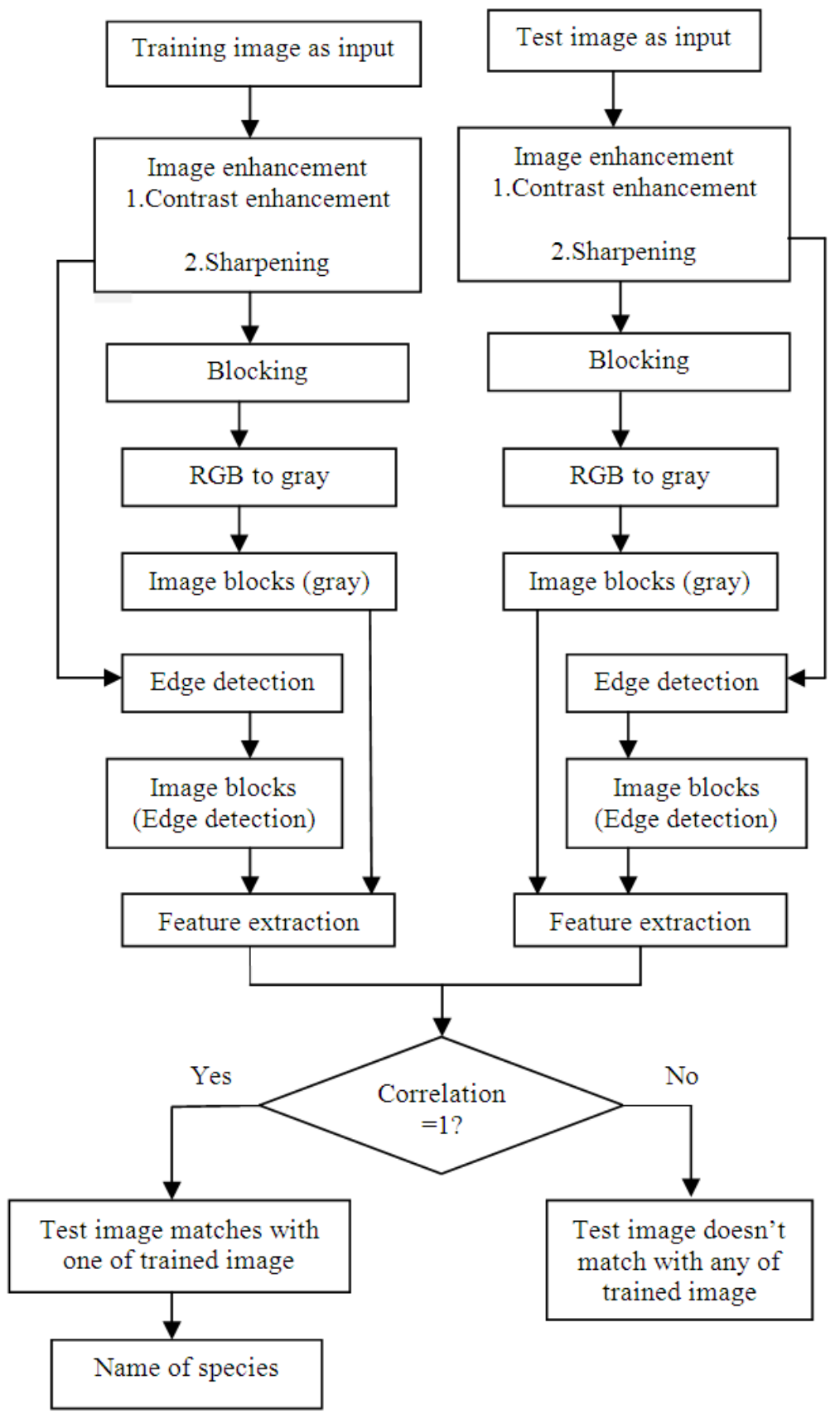

Fig. 1. Proposed wood recognition system

\subsubsection{Contrast Enhancement}

Contrast enhancements improve the perceptibility of objects in the scene by enhancing the brightness difference between objects and their backgrounds. It's a useful method for processing scientific images such as X-Ray images or satellite images. It is also useful to improve detail in photographs that are over or under-exposed. Contrast enhancement processes adjust the relative brightness and darkness of objects in the scene to improve their visibility. 
Table 1. Name of Wood Species

\begin{tabular}{ll}
\hline Name & Scientific name \\
\hline Jack & Artocarpus heterophyllys \\
Rose wood & Dalbergia sissoo \\
Satin & Santalum album \\
Sal & Chloroxylon swietenia \\
Mahogany & shorea robusta \\
Mulberry & Swietenia mahagoni \\
Oak & Broussanetia papyrifera \\
Iron wood & Quercus alnifolia \\
Teak & Carpinus caroliniana \\
\hline
\end{tabular}

\subsubsection{Image Sharpening}

Image sharpening refers to any enhancement technique that highlights edges and fine details in an image. Image sharpening is widely used in printing and photographic industries for increasing the local contrast and sharpening the images.

Human perception is highly sensitive to edges and fine details of an image and since they are composed primarily by high frequency components, the visual quality of an image can be enormously degraded if the high frequencies are attenuated or completed removed. In contrast, enhancing the high-frequency components of an image leads to an improvement in the visual quality.

\subsection{Blocking}

When working with large images, normal image processing techniques can sometimes break down. The images can either be too large to load into memory, or else they can be loaded into memory but then be too large to process. Blocking is the process of dividing the RGB image into several blocks (here four blocks) of equal size. Image blocking method is expected to reduce the number of features used and increase the recognition rate (Tou et al., 2009a).

\subsection{RGB to Gray}

RGB to gray is the process of converting each block RGB image into gray image. This stage is done as needed at a later stage that requires the image of a gray scale. The rgb2gray converts RGB values to grayscale values by forming a weighted sum of the $\mathrm{R}, \mathrm{G}$ and $\mathrm{B}$ components. Also rgb2gray converts RGB images to grayscale by eliminating the hue and saturation information while retaining the luminance (Gasim et al., 2013a).

\subsection{Image Blocks (Gray)}

Image blocks is the images converted from RGB images into gray image, which consists of the full image, top left block, the top right block, bottom left block and the bottom right block.

\subsection{Edge Detection}

Edge Detection reduces the amount of data and filters out useless information, while preserving the important structural properties in the image (Piuri and Scotti, 2010). Edge detection is needed because important features can be extracted from edges of an image. The goals of edge detection are as follows:

- Produce a line drawing of a scene from an image of that scene

- Important features can be extracted from the edges of an image (e.g., corners, lines, curves)

- These features are used by higher-level computer vision algorithms (e.g., recognition)

In this research, the edge detection used is Canny.

\subsection{Canny Edge Detector}

Canny operator is used, because it gives the expected results compared to other operators. Canny aim was to discover the optimal edge detection. Canny algorithm runs in following steps: 1. Smoothing, 2. Finding Gradients, 3. Non-maximum Suppression, 4. Double Thresholding, 5. Edge tracking by Hysteresis.

\subsection{Image Blocks (Edge Detection)}

Image blocks is the images converted from RGB images into gray image, which consists of the full image, top left block, the top right block, bottom left block and the bottom right block (Gasim et al., 2013b).

\subsection{Feature Extraction}

In this study, the wood features are extracted using Gray Level Co-occurrence Matrix (GLCM). It is also referred to as Gray tone spatial dependence matrix. There are a total of 14 features for GLCM. Among the 14 features, 3 features are used in this study to recognize the wood species. The three features are Entropy, Standard deviation and Correlation (Tou et al., 2009b).

\subsection{GLCM}

It is a tabulation of how often different combination of grey levels occur in an image. GLCM calculates how often pairs of pixel with specific values and in a specified values and in a specified spatial relationship occur in an image and creates a matrix and then extracting the statistical features from this matrix. Figure 2 shows a "test image" and the values are image Grey Levels (GLs). 
GLCM texture considers the relation between two pixels at a time, called the reference and the neighbor pixel. In the illustration below, the neighbor pixel is chosen to be the one to the east (right) of each reference pixel. This can also be expressed as a $(1,0)$ relation: 1 pixel in the $\mathrm{x}$ direction, 0 pixels in the y direction.

The top left cell will be filled with the number of times the combination 0,0 occurs, i.e., how many times within the image area a pixel with grey level 0 (neighbor pixel) falls to the right of another pixel with grey level 0 (reference pixel). A different co-occurrence matrix exists for each spatial relationship (above, next to, diagonal). Figure 3 shows the matrix framework for the east $(1,0)$ spatial relationship.

\subsection{Features}

There are a total of 14 features for GLCM. Among the 14 features, 3 features are used in this study to recognize the wood species. The three features are Entropy, Standard deviation and Correlation.

\subsubsection{Entropy}

Entropy is a measure of orderliness (how regular (orderly) the pixel values are within the window). It is a statistical measure of randomness that can be used to characterize the texture of the image. Entropy is usually classified as a first degree measure, but should properly be a $0^{\text {th }}$ degree. The maximum value of ENT is 0.5 . The formula is:

$$
\sum_{i=0}^{G-1} \sum_{j=0}^{G-1} P(i, j) * \log P(i, j)
$$

\subsubsection{Standard Deviation}

It is used to measure the distribution of positive and negative values of a member to the average value of all the members.

To calculate the standard deviation following steps are used:

Calculate the total amount of values of all the pixels of a two dimensional matrix $\mathrm{m} \times \mathrm{n}$ pixels:

$$
\text { Total }=\sum_{i=1}^{m} \sum_{j=1}^{n} \alpha[i, j]
$$
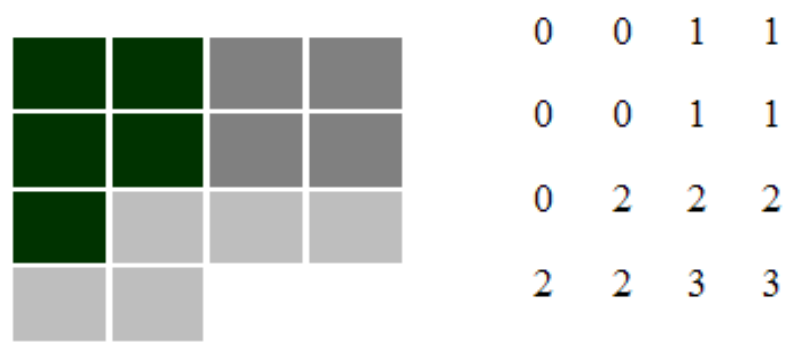

Fig. 2. Test image and its gray values

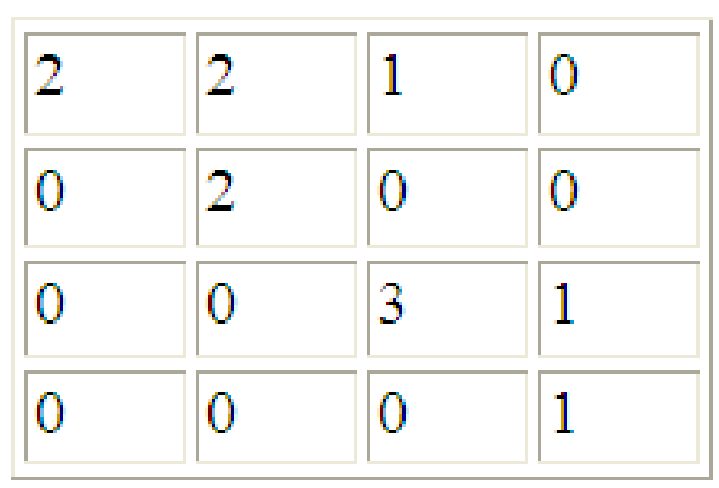

Fig. 3. GLCM values for the given image

Calculate the average values pixel matrix $m \times n$ pixels:

$$
\text { Mean }=\frac{\text { total }}{\mathrm{m} \times \mathrm{n}}
$$

Calculate the standard deviation:

$$
\operatorname{Std}=\sqrt{\frac{1}{(m \times n)-1} \sum_{i=1}^{m} \sum_{j=1}^{n}(a[i, j]-\text { mean })^{2}}
$$

\subsubsection{Correlation}

It is the measure of how a pixel is correlated to its neighbor over the entire image. Range of value for correlation is 0 to 1 :

$$
\text { Correlation }=\sum_{i=1}^{G-1} \sum_{j=1}^{G-1} \frac{\{i * j\} \times P(i, j)-\left\{\mu_{z} \times \mu_{y}\right\}}{\sigma_{z} \times \sigma_{y}}
$$




\subsection{Classification using Correlation}

The correlation texture measures the linear dependency of grey levels on those of neighboring pixels. The correlation is a measure of association (resemblance) between two images to find those portions that match according to the measure of correlation (Khalid et al., 2011). The formula used to find the correlation value is:

$$
\mathrm{R}=\frac{\sum(\mathrm{x}-\operatorname{Avg}(\mathrm{x})) *(\mathrm{y}-\operatorname{Avg}(\mathrm{y}))}{\sqrt{\sum\left(\mathrm{x}-\operatorname{Avg}(\mathrm{x})^{2} * \sum(\mathrm{y}-\operatorname{Avg}(\mathrm{y}))\right.}}
$$

where, $\mathrm{R}$ is a Correlation factor, $\mathrm{x}$ represents features of trained wood image and $\mathrm{Y}$ represents features of testing image. If both images are identical then $\mathrm{R}$ will be 1 .

\section{RESULTS AND DISCUSSIONS}

This research can identify 10 types of Indian wood species. In this research three features are used to recognize the wood that provides the accuracy of more than $95 \%$. The trained images results are stored in the database.

The tested images vales are compared with the values of trained images. If trained images value matches the test images values then the name of wood species will be displayed. For testing we have used five images of each type and so there are a total of 100 images were used for testing. Figure 4 shows that the correlation value of tested image matches with the Sal, the tested image is identified as Sal wood. The Table 2 shows the correlation value of ten wood species.

Table 2. Correlation values of wood species

\begin{tabular}{lr}
\hline Wood species & Correlation value \\
\hline Sal & 0.1918 \\
Satin & 0.8823 \\
Mulberry & 0.7174 \\
Mahogany & 0.2321 \\
Iron wood & 0.6951 \\
Sandal wood & 0.3954 \\
Rose wood & 0.0177 \\
Teak & 0.1545 \\
Oak & 0.5652 \\
Jack & 0.0812 \\
\hline
\end{tabular}

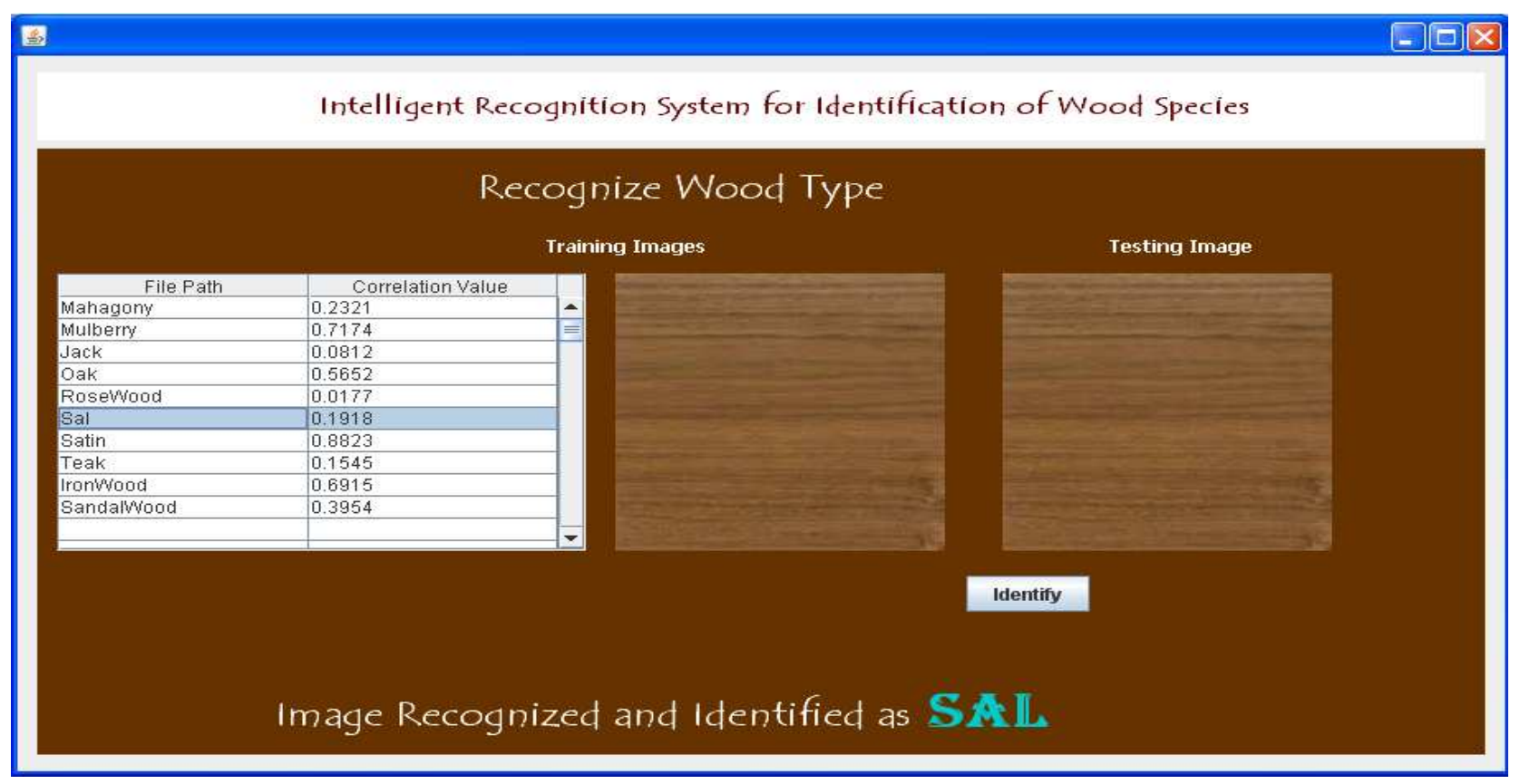

Fig. 4. Recognition system that identifies the tested image as SAL wood 


\section{CONCLUSION}

In this study, an intelligent recognition system for the identification of wood species has been proposed. The system was designed to be cost-effective. The system shows high accuracy in recognizing wood when compared to manual identification. Image processing technique has been used to improve accuracy. GLCM is used as texture classification technique to extract the features of wood. For classifying the wood, correlation technique has been used. The system shows accuracy of more than $95 \%$ in recognizing 10 Indian wood species.

There are still some combinatons of blocks that have not been tested. The system use only three features, we can add features to increase the recognition rate. The design of wood recognition system can be implemented onto an embedded platform that has camera, processing board and LCD display.

\section{REFERENCES}

Ardian, F., 2012. Tropical Wood Texture Classification. 1st Edn., Vibot Day, Girona, Spain.

Bremanath, R., 2009. Wood species recognition system. Int. J. Electrical Comput. Eng., 52: 873-879.

Gasim, A. Harjoko, K.B. Seminar and S. Hartati, 2013a. Image blocks model for improving accuracy in identification systems of wood type. Int. J. Adv. Comput. Sci. Applic., 4: 48-53.

Gasim, A. Harjoko, K.B. Seminar and S. Hartati, 2013b. Merging feature method on RGB image and edge detection image for wood identification. Int. J. Comput. Sci. Inform. Technol., 4: 188-193.

Gurau, L.M.C., M. Timar and F. Porojan, 2013. Image processing method as a supporting tool for wood species identification. Wood Fiber Sci., 45: 1-11.
Hasan, A.F., M.F. Ahmad, M.N. Ayob, S.A.A. Rais and N.H. Saad et al., 2013. Application of binary particle swarm optimization in automatic classification of wood species using gray level co-occurrence matrix and knearest neighbor. Int. J. Sci. Eng. Res., 4: 50-55.

Hazra, D., 2011. Texture recognition with combined GLCM, wavelet and rotated wavelet features. Int. J. Comput. Electrical Eng., 3: 146-150.

Khalid, M., E.L.Y. Lee, R. Yusof and M. Nadaraj, 2008. Design of an intelligent wood species recognition system. IJSSST.

Khalid, M., R. Yusof and A.S.M. Khairuddin, 2011. Tropical wood species reocgnition system based on multi-feature extractors and classifiers. Proceedings of the 2nd International Conference on Instrumentation Control and Automation, Nov. 1517, IEEE Xplore Press, pp: 6-11. DOI: 10.1109/ICA.2011.6130117.

Piuri, V. and F. Scotti, 2010. Design of an automatic wood types classification system by using fluorescence spectra. IEEE Trans. Syst. Man Cybernet., 40 : 358-366. $\quad$ DOI: 10.1109/TSMCC.2009.2039479

Tou, J.Y., Y.H. Tay and P.Y. Lau, 2007. Computer vision-based wood recognition system. Universiti Tunku Abdul Rahman (UTAR), Malaysia.

Tou, J.Y., Y.H. Tay and P.Y. Lau, 2009a. A comparative study for texture classification techniques on wood species recognition problem. Proceedings of the Fifth International Conference on Natural Computation, Aug. 14-16, IEEE Xplore Press, pp: 812. DOI: $10.1109 /$ ICNC.2009.594

Tou, J.Y., Y.H. Tay and P.Y. Lau, 2009b. Rotational invariant wood species recognition through wood species verification. Proceedings of the 1st Asian Conference on Intelligent Information and Database Systems, (DS '09), IEEE Xplore Press, Dong Hoi, pp: 115-120. DOI: 10.1109/ACIIDS.2009.10 